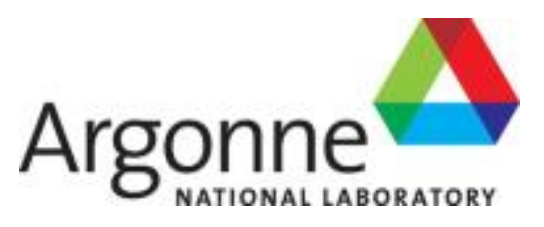

\title{
Shear Ram Verification Test Protocol (VTP) Best Practices
}

Best Practice for Verification Test Protocol of Blowout Preventer (BOP) Shear Rams Used for US OCS Oil and Gas Operation

Global Security Sciences Division 


\title{
Report Authors
}

This report was prepared by the Strategic Alliance for Global Energy Solutions (SAGES) Center team under the direction of Dan M. Fraser.

About Argonne National Laboratory

Argonne is a U.S. Department of Energy laboratory managed by UChicago Argonne, LLC under contract DE-AC02-06CH11357. The Laboratory's main facility is outside Chicago, at 9700 South Cass Avenue, Argonne, Illinois 60439. For information about Argonne and its pioneering science and technology programs, see www.anl.gov.

\section{DOCUMENT AVAILABILITY}

Online Access: U.S. Department of Energy (DOE) reports produced after 1991 and a growing number of pre-1991 documents are available free via DOE's SciTech Connect (http://www.osti.gov/scitech/)

\author{
Reports not in digital format may be purchased by the public from the \\ National Technical Information Service (NTIS): \\ U.S. Department of Commerce \\ National Technical Information Service \\ 5301 Shawnee Rd \\ Alexandria, VA 22312 \\ www.ntis.gov \\ Phone: (800) 553-NTIS (6847) or (703) 605-6000 \\ Fax: (703) 605-6900 \\ Email: orders@ntis.gov
}

Reports not in digital format are available to DOE and DOE contractors from the Office of Scientific and Technical Information (OSTI):

U.S. Department of Energy

Office of Scientific and Technical Information

P.O. Box 62

Oak Ridge, TN 37831-0062

www.osti.gov

Phone: (865) 576-8401

Fax: (865) 576-5728

Email: reports@osti.gov

\section{Disclaimer}

This report was prepared as an account of work sponsored by an agency of the United States Government. Neither the United States Government nor any agency thereof, nor UChicago Argonne, LLC, nor any of their employees or officers, makes any warranty, express or implied, or assumes any legal liability or responsibility for the accuracy, completeness, or usefulness of any information, apparatus, product, or process disclosed, or represents that its use would not infringe privately owned rights. Reference herein to any specific commercial product, process, or service by trade name, trademark, manufacturer, or otherwise, does not necessarily constitute or imply its endorsement, recommendation, or favoring by the United States Government or any agency thereof. The views and opinions of document authors expressed herein do not necessarily state or reflect those of the United States Government or any agency thereof, Arg onne National Laboratory, or UChicago Argonne, LLC.

This report was prepared by Argonne National Laboratory (ANL) under contract to the Department of Energy (DOE) through an interagency agreement between the Department of the Interior, Bureau of Safety and Environmental Enforcement (BSEE) and the DOE. The opinions, findings, conclusions, and recommendations expressed in the report are those of the authors and they do not necessarily reflect the views or policies of BSEE. 
ANL-17/13

\section{Shear Ram Verification Test Protocol (VTP) Best Practices}

Best Practice for Verification Test Protocol of Blowout Preventer (BOP) Shear Rams Used for US OCS Oil and Gas Operation

prepared by

Roy A. Lindley

Joseph C. Braun

Global Security Sciences Division, Argonne National Laboratory

Prepared for the US Department of the Interior, Bureau of Safety and Environmental Enforcement

July 17,2017 


\section{Table of Contents}

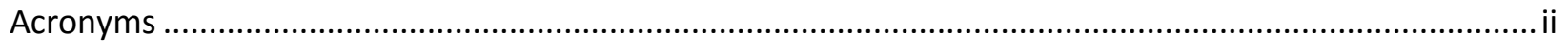

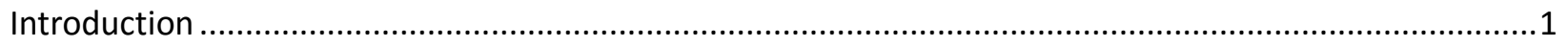

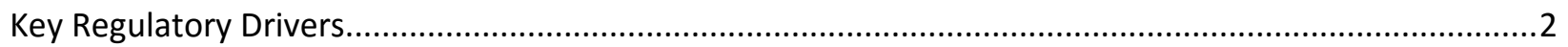

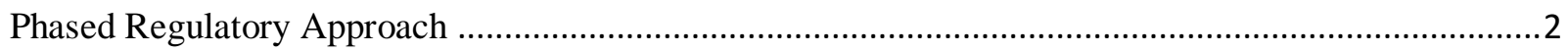

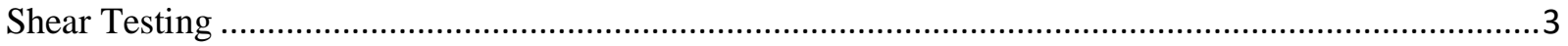

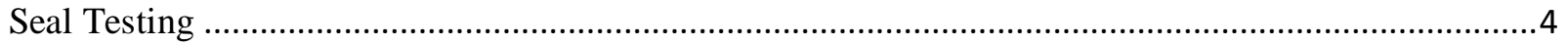

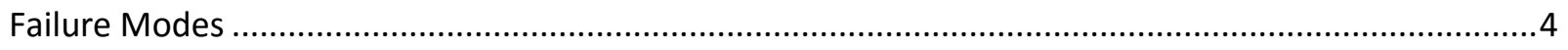

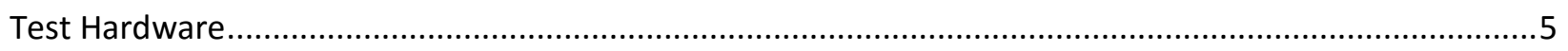

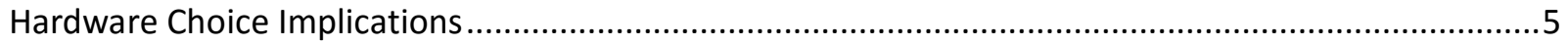

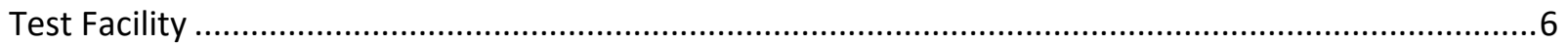

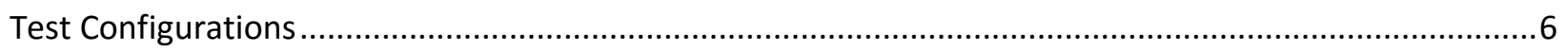

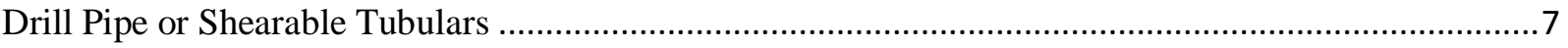

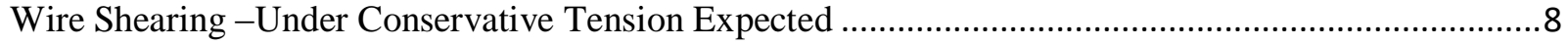

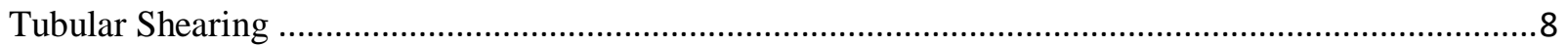

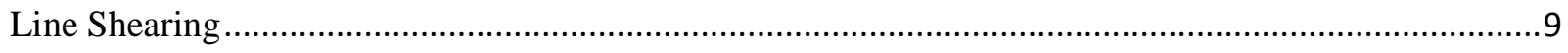

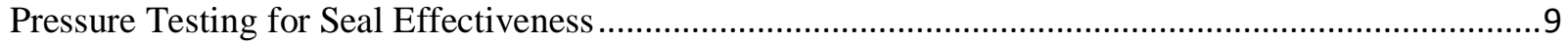

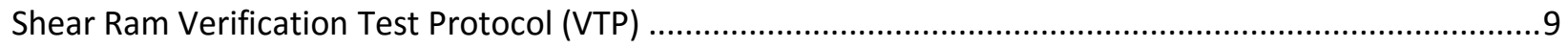

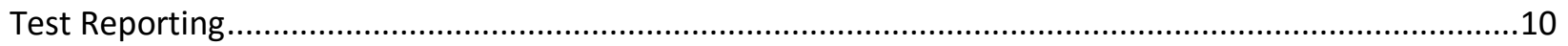

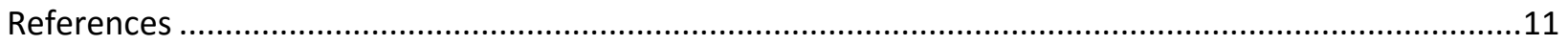

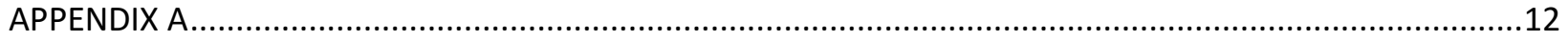

Regulatory Expectations Will Increase Over Time ...........................................................

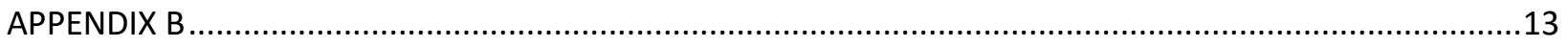

Example of a BSR Verification Testing Protocol ................................................................. 


\section{Acronyms}

\begin{tabular}{|l|l|}
\hline ANL & Argonne National Laboratory \\
\hline ANSI & American National Standards Institute \\
\hline API & American Petroleum Institute \\
\hline BAVO & BSEE-Approved Verification Organization \\
\hline BOP & Blowout Preventer \\
\hline BSEE & Bureau of Safety and Environmental Enforcement \\
\hline BSR & Blind Shear Ram \\
\hline CFR & Code of Federal Regulations \\
\hline DOI & Department of the Interior \\
\hline HPHT & High-Pressure, High-Temperature \\
\hline MASP & Maximum Anticipated Surface Pressure \\
\hline OCS & Outer Continental Shelf \\
\hline OEM & Original Equipment Manufacturer \\
\hline QA/QC & Quality Assurance/Quality Control \\
\hline RWP & Rated Working Pressure \\
\hline TR & Technical Report \\
\hline VTP & Verification Test Protocol \\
\hline
\end{tabular}




\section{$\underline{\text { Introduction }}$}

A blowout preventer (BOP) is a critical component used on subsea oil and gas wells during drilling, completion, and workover operations on the U. S. outer continental shelf (OCS). The purpose of the BOP is to seal oil and gas wells, and in the case of an emergency well-control event, to prevent the uncontrolled release of hydrocarbons.

One of the most important components of the BOP is the hydraulically operated blind shear ram (BSR) that shears drilling-related components, such as drill pipes, casings, tubings, and wirerelated tools that may have been placed in the well. In addition to shearing these components, the BSR must form a seal to keep hydrocarbons within the well bore, even when under the highest well-fluid pressures expected.

The purpose of this document is for Argonne National Laboratory (ANL) to provide an independent view, based on current regulations, and best practices for testing and confirming the operability and suitability of BSRs under realistic (or actual) well conditions. It is hoped that these best practices will positively influence the development of protocols by the industry for testing blind shear rams in the U.S. OCS.

There are many best practices topics. A rational protocol should, at a minimum, address the following topical areas:

- Normal statistical variation of test results. One approach might be specifying a number of shear tests as sufficient. Another is to adopt statistically-based acceptance criteria.

- Define accuracy of pressure measurement as percent of full scale (typically $1 \%$ of full scale).

- Set a minimum data collection frequency during shear phase of testing $(20 \mathrm{~Hz}$ for example).

- Consistently monitor, record, and report critical pressures, volumes, and temperatures during testing, such as both closing and opening and any other pressure, volume or temperature that affects BSR performance.

- Calculate and report shear force at peak closing pressure at moment of shear.

- Specify the shear sample motion constraint (5 degree maximum deflection for example).

- Recognize and consider the consequences of different shear conditions such as "off bottom," "on bottom," and "on bottom and in compression". The BOP system should have the ability to mitigate compression of the pipe stub between the shearing rams when both shear rams are closed per 30CFR 250.734(a)(16)(ii).

- Test shearing performance only on non heat affected or cold worked zones of test samples.

- As part of test preparation, check to verify there is not leakage past ram operator pistons.

- Record and report detailed photographic and video documentation.

- Incorporate concept of "traceability" such as who performed tests, when tests performed, and exactly which pieces of equipment were used (e.g. serial numbers). 
- Adhere to a 30-minute minimum requirement for high and low pressure sealing test time immediately following shear. (This is required by 30CFR 250.732(b)(vi)(ii)).

- Test at the outermost edges of the shear blades of the shear ram positioning system per 30CFR 250.734(a)(16) and 30CFR 250.732(b)(1)(iv). Testing should be conservative and include engineering judgement regarding the worst case(s).

- Include wire line shearing and sealing per regulations.

- Institute full quality control including appropriate notations on data sheets and text.

- Assure testing does not exceed equipment ratings, such as working pressure and hydraulic operator design limitations.

- Confirm that the BSR conforms to manufacturer specifications before and after the shear tests, such as shear blades and ram blocks.

- Document how test conditions compare to anticipated field conditions. This should include details about equipment, procedures, and pipe.

- Third party or BAVO participation is highly desirable and is planned as required (30CFR 250.732).

- Assure data collection and recording is continuous for each test.

- If adequately done, repeatability and reproducibility should be possible.

This document and the attached sample protocol address these points.

Comprehensive shear-ram verification test requirements are currently found within Title 30 Code of Federal Regulations (CFR) Part 250.700, effective as of July 28, 2016. These regulations identify the numbers and types of tests required. Other documents, such as the American Petroleum Institute (API) Standards and Specifications, provide additional good practices for testing of BOPs and BSRs. These documents are formally incorporated by reference in $30 \mathrm{CFR}$ 250 .

In addition to outlining testing requirements, BSEE regulations also describe the documentation and engineering assessments necessary to demonstrate the completeness of a shear ram performance testing program. These regulations establish that the documentation would allow BOP BSR tests to be repeatable and reproducible. The words "repeatable" and "reproducible" are not intended to be interpreted as a physical testing requirement proving either concept - but only that such could be done.

The question of documentation will be addressed in a separate report.

\section{$\underline{\text { Key Regulatory Drivers }}$}

\section{Phased Regulatory Approach}

In U.S. OCS operations, BOP BSR ratings must exceed the maximum anticipated surface pressure (MASP) defined for the drilling operation.

NOTE: Some BOPs are located at the top of a riser, near the water surface or even midway between the surface and sea floor. Other BOPs are located near the sea floor. 
The term MASP in this report means that shear hydraulic pressures from BSR tests are adjusted according to the BOP datum in the field.

BSRs must be able to shear any drill pipe (excluding tool joints, bottomhole tools, and heavyweight pipe or drill collars), workstring, or tubing used in the field with the shear rams. They must also be able to seal well bores subsequent to shearing.

Additionally, starting no later than April 30, 2018, shear capability under MASP will be required for landing strings (for liners or casings); shear subs on a subsea test tree; tubing with exterior control lines/flat packs; and electric, wire, and slick line used in the field with the shear rams. At least one shear ram must be capable of sealing the wellbore after shearing under MASP conditions as defined for the operation. Any nonsealing shear ram(s) must be installed below a sealing shear $\operatorname{ram}(\mathrm{s}){ }^{1}$

Starting no later than May 1, 2023, BOP systems must have centering mechanisms coupled within each shear ram to position pipes within the shear blade area and ensure shearing will occur when activated. ${ }^{2}$

In US offshore operations, shearing requirements within 30 CFR 250 require compliance with several industry standards, such as:

- relevant provisions of API Standard 53 (1),

- American National Standards Institute (ANSI)/API Specification 6A (2),

- ANSI/API Specification. 16A (3),

- API Specification 16C (4),

- API Specification 16D (5),

- ANSI/API Specification 17D (6), and

- $\quad$ API Specification Q1 (7).

The parenthetical reference numbers (1), (2), etc. identify the formal references for each document. These are found in the References section of this document.

These documents are formally incorporated by reference at 30 CFR $\S 250.198$. $^{3}$

\section{Shear Testing}

Relevant regulations have set forth several outcomes that must be satisfied by the physical testing of BSRs. For example, the use of the actual rig BOP blind-shear equipment and drill pipe (or actual or representative surrogates) is highly desired. If alternate equipment is used, this use should be explained and justified. The following elements are essential parts of any satisfactory BSR shear test:

\footnotetext{
${ }^{1} \S 250.734(\mathrm{a})(1)(\mathrm{ii})$

$2 \S 250.734$ (a)(16)(i)

${ }^{3} \S 250.198$ (entire sections, reference list begins at subsection (e))
} 
1. The physical shear testing must be done in a facility that meets generally accepted quality assurance standards.

2. The test should demonstrate that the rig's BOP BSRs will shear the pipe and any electric, wire, and slick-line to be used in the well.

3. The test protocols and analyses should represent recognized engineering practices for ensuring the repeatability and reproducibility of the tests.

4. The test should provide a reasonable representation of field applications, taking into consideration the physical and mechanical properties of the test specimen.

5. Testing must be performed on the outermost edges of the shearing blades of the shear-ram positioning mechanism.

6. The test must demonstrate that the shearing capacity of the BOP equipment is appropriate by using test specimens that have the physical and mechanical properties comparable to the actual drill pipe and other components used in the field.

\section{Seal Testing}

In addition to demonstrating shearing capability, BSR testing must also demonstrate sealing capability with pressure testing. This seal testing should occur immediately after a successful shear. Related requirements include the following:

1. Pressure tests should occur while the tested BSR remains in the closed and locked position.

2. Successful high-pressure tests must be performed at the rated working pressure $(\mathrm{RWP})^{4}$.

3. Blind-shear rams must seal and hold pressure for 30 minutes during each test.

\section{Faillure Modes}

An underlying premise of testing regulations is that BSR tests must represent the conservative or worst-case conditions that could occur for an actual BOP in the field. For example, most shear tests should be conducted under MASP pressure. However, if using a lower test pressure for MASP can be validly justified, then this seems reasonable.

Because BOP designs differ from one another, it is not always clear whether a particular test situation represents a conservative test. This principle applies to pressure effects, temperature effects (such as the thermal expansion of components), and the position of the pipe within the shear ram. One reason for ensuring at least one set of BSR shear tests is performed on the outermost edge of shearing blades 5 is to provide a configuration that many consider to be a worst case (Figure 1). However, because there are different blind-shear ram designs, this predefined situation may not address all critical issues or potential failures of a BSR.

\footnotetext{
${ }^{4} \S 250.732$ (b)(2)(ii)

5 § 250.732 (b)(1)(iv) and § 250.734 (1)(ii).
} 
It is possible that, in some designs, shearing on the outermost edge may not be the bounding case for an item occupying the well bore. For wells in a high-pressure, high-temperature (HPHT) environment, BSEE looks to the BOP original equipment manufacturer (OEM) to evaluate "all reasonable potential modes of failure." ${ }^{6}$ In the future, BSEE will probably seek to review the testing process using a BSEE approved verification organization (BAVO) ${ }^{7}$ when available. This may necessitate that, for a specific design, additional shear testing be done for verification purposes. To this end, a worst-case (bounding) scenario should be identified by the OEM, described, and tested.

\section{Test Hardware}

\section{Hardware Choice Implications}

$\mathrm{BSEE}$ regulations link BSR testing to the actual BOP on the rig and to the need to represent field conditions to the greatest extent possible. This includes considering the compatibility of rig equipment with the BOP BSR and the corresponding BOP BSR used at the test facility.

An onshore test that uses rig BOP equipment, a blind-shear ram from that BOP, tubulars from rig stock, and actual or comparable rig support systems would be viewed as a representative test. This type of test would leave only minimum technical uncertainties. If surrogate, but representative test equipment were used, the differences would need to be explained and justified - showing how surrogate equipment is equivalent to the actual rig equipment.

It may not always be practical to use an identical BOP blind-shear ram for testing. In such instances, historically at least, a similar shear ram is used ${ }^{8}$. Likewise, a given test may use rig inventory tubulars or non-rig tubulars. The more technical differences that exist between the rig

\footnotetext{
$6 \S 250.732$ (c)(2)(i)

${ }^{7} \S 250.732$ (a)(1) -- the BAVO system is under development at BSEE as of July, 2017.

${ }^{8}$ Note: Current BSEE regulations are not absolute about whether the actual rig BOP/shear ram must be used for blind shear shearing and sealing tests. Arguably, an engineering comparison of two similar shear rams (the rig and a test unit) is defensible, if properly done. With few technical differences between test and field conditions, the uncertainty of this approach is relatively low. Examples of applicable regulatory terminology are: "Your BOP system (excluding casing shear) must be capable of closing and sealing the wellbore at all times....." $§ 250.730(a)$; "Verification that: (1) Test data demonstrate the shear ram(s) will shear the drill pipe at the water depth as required in $\S 250.732$. ., $\S 250.731$ (c)(1); "Demonstrates that the BOP will shear the drill pipe and any electric-, wire-, and slick-line to be used in the well, no later than April 30, 2018.."; § 250.732(b)(1)(i); "Demonstrates the shearing capacity of the BOP equipment to the physical and mechanical properties of the drill pipe....", $\S 250.732(\mathrm{~b})(1)(\mathrm{vi})$; and "The blind shear rams must be capable of shearing at any point along the tubular body of any drill pipe...", . § 250.733(a)(1).
} 
and test situations, the more these differences need to be addressed and reconciled relative to the expected performance of the actual BOP BSR in the field.

It falls to the operator to justify, analyze, and adjust BSR shear test results to ensure compatibility with actual rig performance capabilities. Greater differences imply higher uncertainties. This underscores the need to pay attention to and document the details of testing equipment, test samples, and test setup. It also emphasizes the need to take careful measurements of critical components before and after testing. Depending on future regulatory developments, the operator may wish to employ a BAVO (when available) to assist with the documentation process.

\section{Test Facility}

Shear and pressure integrity testing must be performed by a facility that meets generally accepted quality assurance (QA/QC) standards. ${ }^{9}$

Testing in the facility should provide a reasonable representation of field applications. ${ }^{10}$ The regulatory expectation is that the test results, corresponding documentation, and justification of testing differences all lead to results that are repeatable and reproducible under different and explainable test conditions. ${ }^{11}$

\section{Test Configurations}

ANL envisions at least the following four different types of (well) shearing scenarios. There may be other scenarios and there are some future requirements including those discussed in Appendix A. If there is a chance that such other scenarios will be encountered in the field, these too should be confirmed and tested. Four anticipated scenarios are:

1. Conventional pipe per 30 CFR 250.732(b)(1)(iii) and 30 CFR 250.734(a)(1)(ii), but not tool joints, etc.;

2. Tubular objects with slick lines within their diameters;

3. Tubular objects with or without slick lines in their diameters and (after 4/30/18) electric or wire lines outside of their diameters. The wire lines are assumed to have loads and be under (conservative) tension; and

4. Wires only with typical loads and a conservative tension in the wire.

\footnotetext{
${ }^{9} \S 250.732$ (b)(1)(ii)

${ }^{10} \S 250.732$ (b)(1)(iii)

$11 \S 250.732$ (b)(1)(ii)
} 
Each of these scenarios is seen as different from the others, and it is not expected that any two of these scenarios would occur at the same time. Per $\S 250.733$, surface BOPs must have alternate cutting devices to shear wire lines prior to closing the BOP. Section 250.734 addresses the specifics of subsea shearing functions.

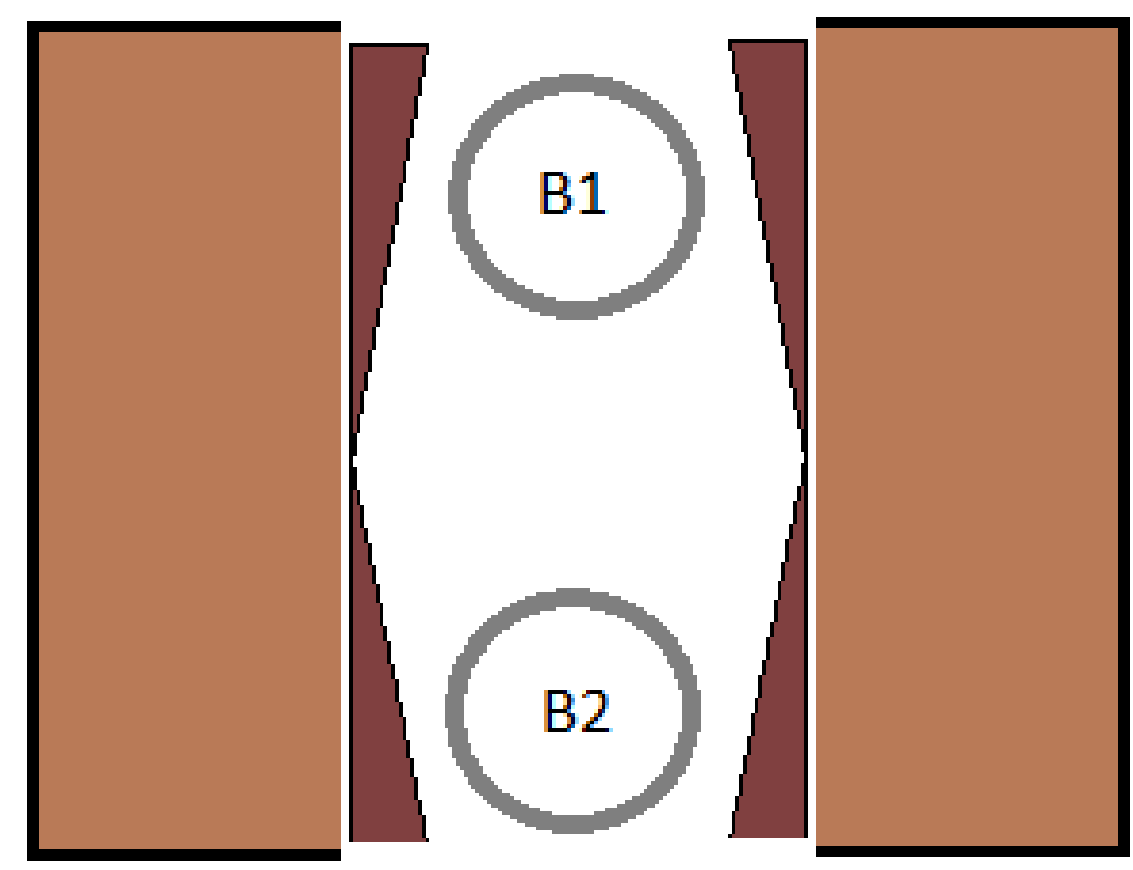

Figure 1 Position of Tubulars for Shear Testing Relative to Shear Blades

\section{Drill Pipe or Shearable Tubulars}

For the shearing of drill pipe or shearable ${ }^{12}$ tubular sections, the test configuration would situate a tubular at the edge of the bore, as shown in Figure 1 (B1 or B2). The tubular is constrained from moving away from the wall (constraint criteria appear in the testing sequence).

If the peak shear pressures between two similar tests fall within acceptance criteria no more similar tests would be required.

The operator may wish to consider the possibility that the tube constrained on the wall is not the worst case situation for shearing a robust tubular. If this is the case, then additional testing is highly encouraged to identify a more appropriate (or conservative) worst case. For example, a best practice, to demonstrate the validity of the test, would shear a pipe that is equivalent in every way to the actual pipe that might be sheared in the field.

\footnotetext{
${ }^{12}$ As defined in the BSEE regulations....for example, drill collars are not shearable tubulars.
} 


\section{Wire Shearing -Under Conservative Tension Expected}

Wire-shearing tests would demonstrate cutting and sealing of representative electric-, wire, or slick lines in the bore. Slick lines are used inside of tubulars, and it is expected that they will shear when the tubular is sheared. The testing should confirm this.

After April 30, 2018 BSRs will be required to shear any wire lines present in the cavity, along with any tubulars that are present. The ability to do this will depend on the detailed design of the BSR and the tension of the wire line. If conservative wire-line tensions are used, a successful test should provide assurance that the BSR will function as needed in the field. This also implies that an alternate set of shear blades may be dedicated to this role; this is recognized for a surface BOP. ${ }^{13}$ Whatever the case, the test should be performed with the device or combination of devices committed to this purpose.

\section{Tubular Shearing}

A single test shearing of a tubular provides one set of data, which is normally compared to a prior peak shear force calculation. However, a single test may shear a pipe but other than comparison to a calculation such a test provides limited information as to whether the results for a specific situation are near average or substantially above or below the average. Theoretically, engineering calculations are validated with data but uncertainty remains. Does that calculation represent an average value or above average value, how much is that value above average, and is the dataset comparable to the current test situation. Recognition and quantification uncertainties in these two areas is important since these results and comparisons are used to evaluate whether the BOP system will shear items on the well bore as intended. The primary technical issue is how best to reduce such uncertainties. Improving calculations is one approach and additional or repeated physical testing for a specific situation is another.

Experience with test results that use the same equipment, sheared items, and procedures will provide a set of data that can be viewed statistically. Any group of tests will have a mean (or average) value, and a standard deviation. Assuming a normal distribution, plus or minus one standard deviation from the mean provides a $68 \%$ confidence interval and, similarly, two standard deviations increases this to $95 \%$. Thus, for a desired level of conservatism, the combination of average and standard deviation define balance of system requirements with a degree of certainty.

Physical shear testing is expensive and time consuming thus normally there should not be an expectation for an applicant to conduct a statistically significant number of tests for each situation. In the normal process of testing, if the results of any given test versus calculation comparison fall outside of a normally expected variations by more than one standard deviation, then tests or calculations should continue until two or more successive results fall within that

\footnotetext{
${ }^{13} \S 250.733(a)(1)$
} 
band. Changes to the test arrangement to achieve these results should be documented and be incorporated in periodic calculation validation updates.

Until a statistical database (at least 50-100) has been developed and used to validate calculation models, a difference of successive test results that fall within $10 \%$ of one another and are below calculated predictions is considered to be reasonable ${ }^{14}$. This approach necessitates at least two tests of each shearing situation and, depending on the resulting comparisons, may require three or more tests. Obviously the second test provides added assurance that the first test is not an extreme value for some unexplained reason.

\section{Line Shearing}

The BSR should shear all lines present within the well bore, along with the drill pipe or tubulars in the BSR. Lines can be inside or outside of the tubulars and test scenarios should consider these possibilities. Independent of the methodology used in any particular BSR, the shear of any line must be complete. Lines should be under a conservative tension during the shearing phase of the shear and seal test. (A conservative tension would be lower than that expected in the field.)

\section{Pressure Testing for Seal Effectiveness}

Following a shear test, a BSR is required to seal the well bore. According to regulations, this means the BSR holds rated working pressure (RWP) for 30 minutes. ${ }^{15}$

If a wire line is present inside a tubular, does not shear, and prevents a successful sealing test, then the shearing and sealing test should be repeated until a successful sealing test can be achieved. For the shear of a wire in a tubular, it is expected that a pressure seal test would be conducted post shear with the BSR in the closed-and-locked position.

\section{$\underline{\text { Shear Ram Verification Test Protocol (VTP) }}$}

Appendix B presents a Sample or Example of a Test Protocol. The intent of this Example is to demonstrate the level of detail that may be needed to ensure that tests can be performed on the same components at different facilities and in different devices in such a manner as to produce the same or very similar results. These considerations include not only the manner in which the test is performed, but also the information that is recorded and reported. The goal is to develop methods that can be repeated across the industry, and provide confidence that the on-shore testing is conservative with regard to the actual shearing that may need to occur in the field.

\footnotetext{
${ }^{14}$ Random variation leads to possible differences in measured values. These are usually expressed as the term $(\sqrt{n} / n)$, such that random variations for 100 samples is $10 / 100= \pm 10 \%( \pm 1 \%$ for 10,000$)$. Thus, based on an assumed database of about 100 tests, a value of about $+/-10 \%$ between any two samples is judged to be reasonable. Ref: George, E. P. et. al, Statistics for Experimenters, Wiley and Sons, 1978.

$15 \S 250.732$ (b)(2)(ii)
} 


\section{Test Reporting}

BSEE is preparing separate guidance for reporting test results. This separate guidance covers documentation of test results, photographs or videos, description of the test facility, shear pressure prediction calculations, engineering comparisons with rig equipment and capabilities, and MASP adjustments, etc. 


\section{$\underline{\text { References }}$}

1. American Petroleum Institute. Blowout Prevention Equipment Systems for Drilling Wells. API Standard 53, Fourth Edition. November 2012.

2. American Petroleum Institute (ANSI/API). Specification for Wellhead and Christmas Tree Equipment. ANSI/API Specification 6A, Nineteenth Edition, July 2004; Errata 1 (September 2004), Errata 2 (April 2005), Errata 3 (June 2006) Errata 4 (August 2007), Errata 5 (May 2009), Addendum 1 (February 2008), Addenda 2. December 2008.

3. American Petroleum Institute (ANSI/API). Specification for Drill-through Equipment. ANSI/API Specification 16A, Third Edition Reaffirmed. August 2010.

4. American Petroleum Institute (ANSI/API). Specification for Choke and Kill Systems, First Edition. Specification 16C, Reaffirmed. July 2010.

5. American Petroleum Institute. Specification for Control systeems for Drilling Well Control Equipment and Control Systems for Diverter Equipment. Specification 16D, Second Eidtion, Reaffirmed. August 2013.

6. American Petroleum Institute (ANSI/API). Design and Operation of Subsea Production SystemsSubsea Wellhead and Tree Equpment. ANSI/API Specification 17D, Second Edition. May 2011.

7. - Specification for Quality Programs for the Petroleum and Natural Gas Industry. ANSI/API Specification Q1, Eighth Edition with Addendum 1. June 2010.

8. - Specification for Wellhead and Christmas Tree Equipment. ANSI/API Specification 6A, Nineteenth Edition with addenda 2, 3, and 4. December 2008.

9. - Specification for Quality Programs for the Petroleum and Natural Gas Industry. ANSI/API Specification Q1, Eighth Edition with Addendum 1. June 2010. 


\section{$\underline{\text { APPENDIX A }}$}

Regulatory Expectations Will Increase Over Time

Currently 30 CFR 250.730 requires that (the):

"BOP system (excluding casing shear) must be capable of closing and sealing the wellbore at all times, including under anticipated flowing conditions for the specific well conditions, without losing ram closure time and sealing integrity due to the corrosiveness, volume, and abrasiveness of any fluids in the wellbore that the BOP system may encounter," and

30 CFR 250.732(b)(1) that states that:

"Prior to beginning any operation requiring the use of any BOP", the operator "must submit verification by a BSEE approved verification organization (BAVO) and supporting documentation as required" to the appropriate District Manager and Regional Supervisor.

In US OCS operations, shear-ram performance should have been proven no later than July 28 , 2016 under maximum anticipated surface pressure (MASP) for any drill pipe, workstring, and tubing to be used in the field with the shear rams. ${ }^{16}$

Additionally, starting no later than April 30, 2018, shear capability under MASP will be required for landing strings (for liner or casing); shear subs on a subsea test tree; tubing with exterior control lines/flat packs; and electric, wire, and slick line used in the field with the shear rams ${ }^{17}$.

Furthermore, starting no later than May 1, 2023, BOP systems will have a "mechanism coupled with each shear ram to position the entire pipe, completely within the area of the shearing blade and ensure shearing will occur any time the shear rams are activated." 18

Note that, in US offshore operations, shearing requirements within 30 CFR 250 require compliance with industry standards, such as relevant provisions of:

API Standard 53, ANSI/API Spec. 6A, ANSI/API Spec.16A, API Spec. 16C, API Spec. 16D, ANSI/API Spec. 17D, and API Spec. Q1, -as specified in 30 CFR 250.198.

\footnotetext{
${ }^{16}$ Paraphrased from $\S 250.733(\mathrm{~b})(2)$

${ }_{17}$ Paraphrased from $\S 250.734$ (1)(ii)

${ }^{18}$ At § 250.734(16)(i)
} 


\section{$\underline{\text { APPENDIX B }}$}

Example of a BSR Verification Testing Protocol

\section{Shear Ram Verification Test Protocol (VTP) ${ }^{19}$}

As a minimum, the following elements are expected to be present in an acceptable testing protocol or procedure:

\section{Equipment Inspection and Characterization}

An inspection should be completed prior to shear testing to ensure that the tested shear ram is fully characterized and that process should be coordinated with the chosen BSEE Approved Verification Organization (BAVO) ${ }^{20}$. The characterization should be documented and recorded in a standard manner. A post-test inspection should be performed to confirm there is no change or to document a change that is attributable to the shear testing. If damage has occurred during testing and repairs are necessary, a thorough inspection and documentation should occur before testing continues. The following elements are typically found in a good protocol:

1. Inspection of the interior and exterior of the shear ram assembly, inclusive of all components, to verify conformance with original equipment manufacturer (OEM) specifications. This includes recording dimensions and surface conditions, and confirming part numbers.

2. Photograph and record configuration and dimensional aspects, particularly those of the BOP wellbore and ram blades.

3. Record manufacturer's details such as models, serial numbers, part numbers, etc.

4. Setup and document appropriate test facility and equipment including calibration of instrumentation, frequency of measurements, redundancy, etc...

\footnotetext{
19 In all cases the protocol and testing process shall comply with the testing and information required in applicable BSEE regulations. Should there be a conflict with this document and the regulations, the regulations prevail. ${ }^{20}$ Or prior to full implementation of the BAVO, an independent third party.
} 


\section{Physical Test Process $^{21}$}

\section{Characterize Unloaded Blade Motion Resistance}

1. With the BSR bore initially empty and free of debris, fill the bore with test fluid (water, water with additives, etc.) to the level above the shear ram blade plane as anticipated for testing as specified by the BOP OEM.

2. Adjust the hydraulic fluid supply pressure regulator for the shear ram closing chamber equivalent to the lesser of the maximum rated working pressure of the BOP hydraulic system or no greater than the OEM recommended pressure for shearing.

3. To establish a ram block motion functional baseline, close and open the rams at least three

(3) times while recording both the close and open side pressures and volumes.

- Tests for non-high pressure high temperature (HPHT) service may be performed at ambient temperature.

- For HPHT service environment $\left(\geq 15,000 \text { psi or } \geq 350^{\circ} \mathrm{F} \text { at the wellhead }\right)^{22}$, baseline function testing should be considered at rated conditions. (While advisable, technically maintaining HPHT service conditions during shear testing is not a regulatory requirement). There are requirements for the BAVO to conduct a comprehensive review of the equipment for service condition.)

NOTE: Before initiating any shear tests, a function test should confirm that the pressure required to close/open rams remains within the range of the first three function tests. If not within range, then two additional function tests should be performed to establish a new baseline.

a) Close/Open cycles should be performed using the maximum hydraulic operating pressure for closing and the OEM recommended pressure for opening.

b) Both the close and open side pressures and time should be continuously recorded as of start of baseline function testing of the rams along with any other critical pressures, temperatures, and volumes.

\section{Select and Prepare Test Specimen(s)}

4. Select and thoroughly document a shearing sample (specimen) that is representative of the rig pipe, tubing, and/or line to be sheared. The specimen should have comparable outside/inside diameter, maximum wall thickness, hardness, and other characteristics to the actual item(s) to be used in the well operation.

a) Section the sample into manageable lengths. For example, drill pipe should be a minimum of $1.2 \mathrm{~m}$ (4 ft.) in length (as stated in API 16A, Third Edition (3)), however

\footnotetext{
${ }^{21}$ Testing organization is expected to establish and follow safe practices during testing.

$22 \S$ CFR 250.732(c)(3)
} 
it may be longer when multiple shear tests are planned on the same specimen. The length must be adequate to assure that:

i. Any cold- or hot-work affected areas of the specimen are outside of the specimen collapse or deformation zone which is caused by shearing (see Figure 2);

ii. Specimen material sample testing and the related collapse or deformation areas occurs between such cold- or hot-work affected areas; and

iii. Guides do not restrict any collapse deformation of the specimen.

b) Above and below the plane on the test specimen where shearing is to occur and some distance (typically 1 pipe diameter or a few inches in the case of line) above and below the expected point of contact with the shear ram blades, characterize the specimen (wall thickness, eccentricity, outside diameter, hardness). Mark and label the specimen shear plane and the upper and lower sections for post-test traceability.

c) Mark tubular specimens circumferentially at the points closest to the shear rams where the centering/restraining guide(s) will hold the specimen and label as such.

\section{Position Test Specimens in Test Bore}

5. Drain and Clear BSR test bore.

6. Lower Test Specimen and Associated Hardware into BSR Bore.

a) Mount and suspend the specimen vertically above the BSR such that azimuthal orientation of any wall thickness or eccentricity at the shear plane represents the highest resistance to shear (highest shear force for the item being sheared)

b) Position the specimen so that there is sufficient space for the lower part of the specimen to drop after shearing for any unrestrained test.

7. Apply the following for the particular specimen geometry being tested:

a) For regulatory required Restrained Outermost Compression (Side-Load) test, report how the compression/side-load is applied, the amount of side-load applied (measured or calculated) during the test up to the point in time when the specimen does shear, and how the test sample is restrained above and below the shear plane. The physical application of the compression/side-load should not contact any collapse deformation of pipe.

b) For an Unrestrained Centered with Guides test, the specimen should be guided above and below the shear plane such that the specimen cannot deflect more than $5^{\circ}$ in the direction of the ram operator axis (see Figure 1). The following applies: 
i. The method of guiding the pipe should not restrict any collapse deformation of pipe;

ii. The location of the centering guide relative to the shear plane should be fully documented;

iii. The type of guide should be fully documented.

c) For Unrestrained Diametrically Off-Center / Outermost Perpendicular to Axis Outermost tests, there should be no guide that would otherwise restrict the item position or deflection.

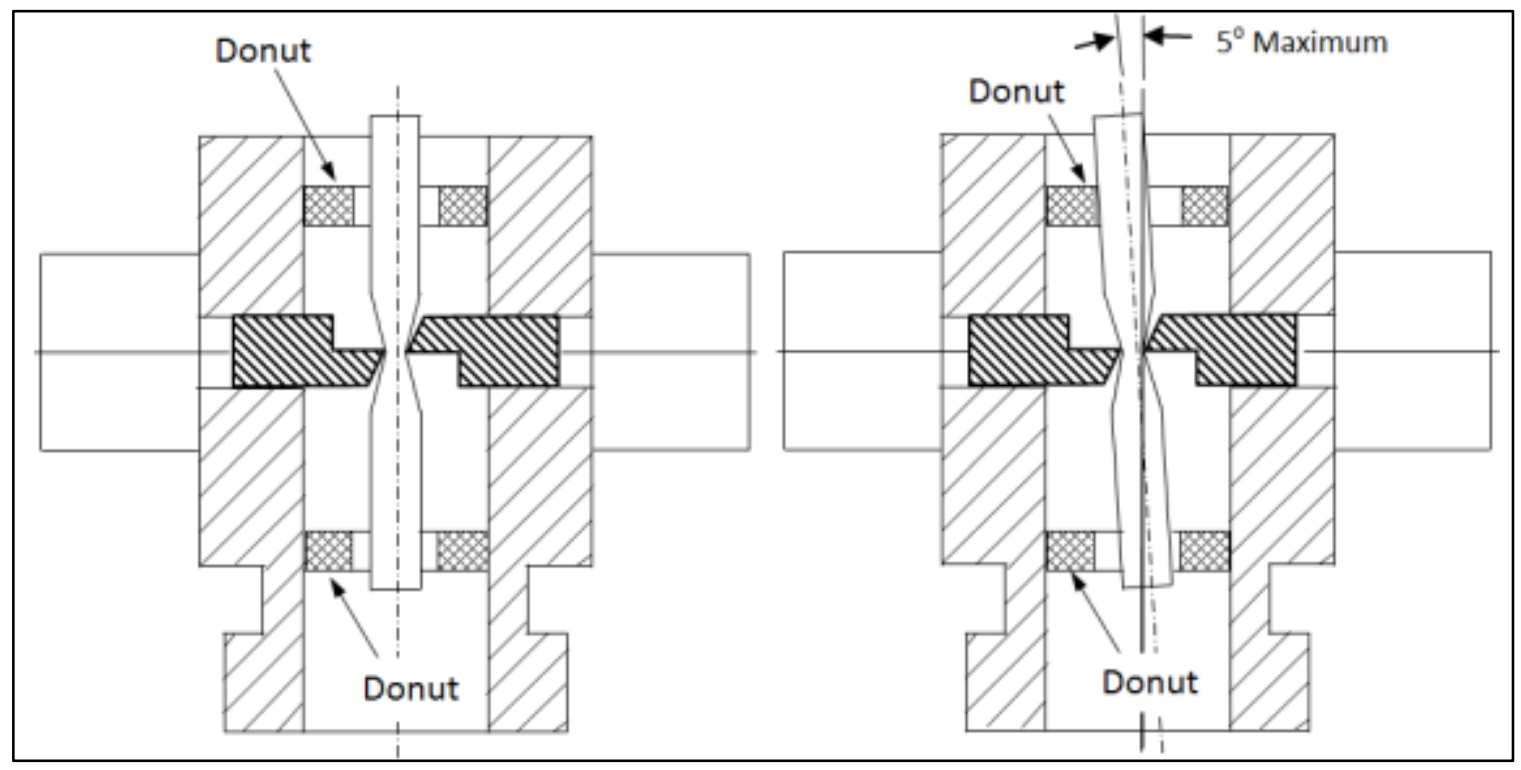

Figure 1 Centered Shear Pipe Setup

\section{Proceed with Physical Shear Test}

8. Photograph the interior of the test set-up once the pipe sample is positioned for shearing to illustrate the physical alignment and positioning of the pipe or line within the bore.

9. Fill BSR Bore with test fluid and achieve initial conditions of pressure or temperature as applicable for shearing.

10. Start the data acquisition system to record test pressures and temperatures at a rate equal to or greater than $20 \mathrm{~Hz}$ (20 scans per second). 
11. Function the control valve to close the shear rams in one continuous operation (no stoppage of the blade travel other than the natural reduction of travel speed that may occur when the blades first contact the specimen) and within the maximum allowed response time specified in API 16D for performing the shearing and sealing function action. Once the shear is complete, the shear rams should remain closed and in the locked position until after the pressure integrity/sealing tests are complete for the BSRs. Data acquisition may be reduced to recording at $1 \mathrm{~Hz}(1$ scan per second) after the rams are locked closed.

12. Continue recording pressure in the sealed cavity for 30 minutes.

13. Drain BSR bore and document (photograph) post-test circumstances.

14. Remove and document (photograph) test specimens.

15. Conduct and document the post-test inspection to confirm there is no change, or document a change that is attributable to the shear testing. If damage has occurred during testing and repairs are necessary, a thorough inspection and documentation should occur before testing continues (relative to OEM specifications).

\section{Repeat Tubular Tests}

For both steps 16 and 17, repeat close/open function of the rams remains within the range of the first three function tests. If not, re-establish with 3 new functions is advisable.

16. At this step there is either an initial shear test for a given tubular component (steps 4 through 15) or a repeat shear test for the same tubular component (steps 7 through 18). If the results of the initial shear test of large tubular and the results of the repeat shear test of the same tubular component are within $10 \%$ of each other, then the pressure acceptance criteria is satisfied. ${ }^{23}$ If not repeat steps 4 through 15 until acceptance criteria is satisfied. Additional repeat tests for the same tubular component may be conducted if desired.

17. Shear testing with a different specimen (initial and repeat(s)) or positioning situation should begin at step 4 and proceed to step 15. As per step 16, if such tubular shear test results are within $10 \%$ of each other, then no more such tubular shears are necessary for the test situation.

\section{Wire Shear and Seal Tests}

18. Beginning at step 4 conduct wire shear and seal test(s) marking the wires as appropriate to identify elements after shear has occurred. Repeat if acceptance criteria not satisfied.

${ }^{23}$ Or other reasonable and prudent acceptance criteria. 
Wires that are, or may be, in the bore at the time that the tubular is in the bore, should be tested along with the tubular by completing all steps 4 through 15 . It is important to

confirm that the presence of wires in the bore does not prevent the BSR from sealing and holding pressure as intended.

\section{Special HPHT Testing and Certification}

The HPHT shear test can be done in a similar manner to the foregoing tests, but the pressure and temperature conditions for shearing and sealing in the well should be considered and addressed in the test. Note: An additional BAVO certification is required that verifies the BOP is suitable for the specific HPHT environment, has not been compromised or damaged from previous service, and BOP will operate in conditions in which it will be used. ${ }^{24}$

\section{Documentation}

Results of the forgoing testing shall be thoroughly documented. Thorough documentation would allow a disinterested party to repeat the testing with the expectation of closely reproducing the results. This concept appears in the regulations. A separate document will illustrate appropriate documentation.

$24 \S$ CFR 250.731 (d) (1, 2 and 3) 


\section{Argonne}

\section{Global Security Sciences Division}

Argonne National Laboratory

9700 South Cass Avenue, Bldg. 221

Argonne, IL 60439

www.anl.gov 